\title{
In-plane electronic anisotropy resulted from ordered magnetic moment in iron-based superconductors
}

\author{
S.-F. Wu, ${ }^{1, *}$ W.-L. Zhang, ${ }^{1, \dagger}$ V. K. Thorsmølle, ${ }^{1}$ G. F. Chen, ${ }^{2}$ G. T. Tan, ${ }^{3}$ P. C. Dai,${ }^{4}$ Y. G. Shi, ${ }^{2}$ C. Q. Jin, ${ }^{2}$ T. Shibauchi, ${ }^{5}$ \\ S. Kasahara, ${ }^{6}$ Y. Matsuda, ${ }^{6}$ A. S. Sefat,${ }^{7}$ H. Ding, ${ }^{2}$ P. Richard,${ }^{8}$ and G. Blumberg ${ }^{1,9, \#}$ \\ ${ }^{1}$ Department of Physics and Astronomy, Rutgers University, Piscataway, New Jersey 08854, USA. \\ ${ }^{2}$ Beijing National Laboratory for Condensed Matter Physics, and Institute of Physics, Chinese Academy of Sciences, Beijing 100190, China \\ ${ }^{3}$ Center for Advanced Quantum Studies and Department of Physics, Beijing Normal University, Beijing 100875, China \\ ${ }^{4}$ Department of Physics and Astronomy, Rice University, Houston, Texas 77005, USA \\ ${ }^{5}$ Department of Advanced Materials Science, University of Tokyo, Kashiwa, Chiba 277-8561, Japan \\ ${ }^{6}$ Department of Physics, Kyoto University, Sakyo-ku, Kyoto 606-8502, Japan \\ ${ }^{7}$ Materials Science \& Technology Division, Oak Ridge National Laboratory, Oak Ridge, Tennessee 37831, USA \\ ${ }^{8}$ Institut quantique, Université de Sherbrooke, 2500 Boulevard de l'Université, Sherbrooke, Québec J1K 2Rl, Canada \\ ${ }^{9}$ National Institute of Chemical Physics and Biophysics, 12618 Tallinn, Estonia
}

(Received 5 December 2017; revised 30 June 2020; accepted 7 July 2020; published 24 July 2020)

\begin{abstract}
We study the in-plane electronic anisotropy in the parent compounds of several families of Fe-based superconductors $\left(\mathrm{BaFe}_{2} \mathrm{As}_{2}, \mathrm{EuFe}_{2} \mathrm{As}_{2}, \mathrm{NaFeAs}, \mathrm{LiFeAs}, \mathrm{FeSe}\right.$, and $\left.\mathrm{LaFeAsO}\right)$ by polarization-resolved Raman scattering. We measure intensity of the fully symmetric $c$-axis vibration of As atom mode in the $X Y$ scattering geometry and notice that the mode's intensity is significantly enhanced below the magnetostructural transition only for compounds showing magnetic ordering. In particular, we find that the intensity ratio of this As phonon in the $X Y$ vs. $X X$ scattering geometries is proportional to the square of the ordered magnetic moment. We relate this As phonon intensity enhancement below the Néel temperature in iron pnictides to in-plane electronic anisotropy induced by the collinear spin-density wave order.
\end{abstract}

DOI: 10.1103/PhysRevResearch.2.033140

The lattice, orbital, and magnetic degrees of freedom are strongly coupled in the Fe-based superconductors. This is best evidenced by the observation, in most parent compounds, of a magnetic transition from paramagnetic to collinear antiferromagnetic (AFM), occurring at a temperature $T_{N}$ slightly below the temperature $T_{S}$ at which a structural transition from tetragonal to orthorhombic phase occurs. The interplay between these degrees of freedom is complex and led to a chicken-egg problem for which there is still no consensual view [1,2]. The electronic structure is directly affected by an electronic band folding accompanied by the formation of a collinear spin-density wave (SDW) gap [3-6]. As a result, a significant electronic anisotropy was found for properties measured along the two planar orthogonal Fe-Fe directions (Fig. 1) below the magnetostructural transition, notably in electrical transport [7], optical conductivity [8,9], thermopower [10], local

\footnotetext{
*sw666@ physics.rutgers.edu

${ }^{\dagger}$ Present address: Department of Engineering and Applied Sciences, Sophia University, 7-1 Kioi-cho, Chiyoda-ku, Tokyo 1028554, Japan.

${ }^{\ddagger}$ girsh@ physics.rutgers.edu

Published by the American Physical Society under the terms of the Creative Commons Attribution 4.0 International license. Further distribution of this work must maintain attribution to the author(s) and the published article's title, journal citation, and DOI.
}

density-of-states (DOS) imaging [11], and quasiparticle band dispersions [12].

Raman scattering offers a unique way to study the electronic anisotropy below the magnetostructural transition of the iron-based superconductors [6,13]. For example, one can study the interband transitions along the two planar orthogonal Fe-Fe directions (Fig. 1) in a detwinned sample [6] or investigate the As fully symmetric phonon in a twinned sample [14].

In relation to this study, it has been noticed that the Raman coupling vertex to the As fully symmetric phonon: the $c$-axis vibration of As atom, which modulates the Fe-As-Fe bond angle of the Fe-As tetrahedra (Fig. 1), - is forbidden for $X Y$ scattering geometry in the tetragonal phase, whereas the coupling becomes finite in the orthorhombic phase. However, because orthorhombicity of the lattice constants $\delta$ for all studied materials is weak, the emerging As mode's intensity due to geometrical lattice anisotropy is expected to be small. Surprisingly, a significant intensity of the As phonon in the nearly forbidden $X Y$ scattering geometry has been observed for $\mathrm{Ba}\left(\mathrm{Fe}_{1-x} \mathrm{Au}_{x}\right)_{2} \mathrm{As}_{2}$ below $T_{N}$, in contrast to the weak signals at temperatures between $T_{S}$ and $T_{N}$ [14]. Similar results were reported for $\mathrm{Ba}\left(\mathrm{Fe}_{1-x} \mathrm{Co}_{x}\right)_{2} \mathrm{As}_{2}$ [15]. In addition, the temperature dependence of integrated As phonon intensity was reported to be proportional to square of the magnetic moment $M(T)^{2}$ below $T_{N}$ [14]. The origin of this anomalous intensity enhancement was related to the in-plane electronic anisotropy induced by the collinear SDW order 
(a)

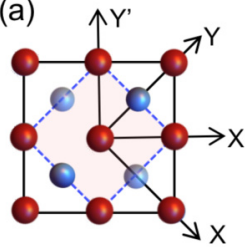

(b)

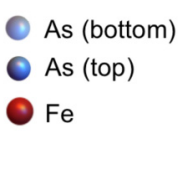

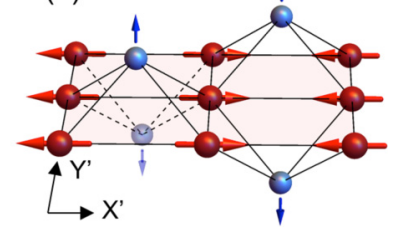

FIG. 1. (a) Definition of the crystallographic directions in the tetragonal 2-Fe unit cell above $T_{S}$ (light red shaded area) and 4-Fe orthorhombic magnetic unit cell below $T_{N}$ (black solid lines). (b) Schematic diagram of the magnetic structure. Red arrows: $\mathrm{Fe}$ local moments forming collinear AFM order. Blue arrows: $c$-axes vibrations of the fully symmetric As phonon mode.

[14]. Furthermore, the Fano model analysis of data revealed that temperature dependence of the Raman vertex amplitude is proportional to magnitude of the magnetic order parameter $M(T)$ [14]. While previous Raman study established the intimate link between the As phonon intensity, the electronic anisotropy and the magnetic order parameter by studying the temperature and doping dependence of the As phonon in the 122 system [14], a detailed study of this relation among different families of iron-based superconductors where the ordered magnetic moment varies is still lacking.

In this paper, we employ polarization resolved Raman scattering spectroscopy to study the in-plane electronic anisotropy for typical 122, 111, 1111, and 11 families of Fe-based superconductors. For all compounds showing magnetic ordering, we observe a strong intensity for the fully symmetric As mode appearing below $T_{N}$ in the nearly forbidden $X Y$ scattering channel as a result of significantly enhanced anisotropy of the in-plane electronic polarizability, while no such enhancement is found for compounds without magnetically ordered state. We also find that the ratio of the As phonon intensity in the $X Y$ vs. $X X$ scattering geometries, $I_{X Y} / I_{X X}$, is proportional to the square of the magnetic ordered moment $M^{2}$. This conclusion is consistent with previous study for $\mathrm{Ba}\left(\mathrm{Fe}_{1-x} \mathrm{Au}_{x}\right)_{2} \mathrm{As}_{2}$ alloys [14]. Because the geometrical lattice anisotropy $\delta=$ $(a-b) /(a+b)$ below $T_{S}$ is relatively small, we conclude that the intensity enhancement of the As phonon in the magnetic phase originates from the collinear SDW order-induced inplane electronic anisotropy.

Single crystals of materials listed in Table I were grown as described in Refs. [16-21]. The corresponding structural phase transition temperature $\left(T_{S}\right)$ and magnetic phase

TABLE I. Summary of $T_{S}, T_{N}$ (in Kelvin), lattice orthorhombicity $[\delta=(a-b) /(a+b)]$, intensity ratio of $A_{g}$ phonon in $X Y$ vs. $X X$ geometries, and ordered magnetic moment/Fe M (in $\mu_{B}$ ) for compounds studied in this paper.

\begin{tabular}{lcccc}
\hline \hline Sample & $T_{S} / T_{N}$ & $\delta(\%)$ & $I_{X Y} / I_{X X}$ & $\mathrm{M}$ \\
\hline $\mathrm{EuFe}_{2} \mathrm{As}_{2}[16]$ & $175 / 175$ & $0.5[22]$ & 3.3 & $0.98[23]$ \\
$\mathrm{BaFe}_{2} \mathrm{As}_{2}[16]$ & $135 / 135$ & $0.4[24]$ & 3.1 & $0.87[25]$ \\
$\mathrm{LaFeAsO}[19,20]$ & $155 / 137$ & $0.24[20]$ & 0.54 & $0.36-0.6[25]$ \\
NaFeAs [18] & $55 / 40$ & $0.18[26]$ & 0.16 & $0.09[25]$ \\
FeSe [21] & $90 /-$ & $0.25[27]$ & 0.017 & - \\
LiFeAs [28] & $-/-$ & 0 & 0 & - \\
\hline \hline
\end{tabular}

transition temperature $\left(T_{N}\right)$ are summarized in Table I. Raman measurements on $\mathrm{BaFe}_{2} \mathrm{As}_{2}, \mathrm{NaFeAs}, \mathrm{EuFe}_{2} \mathrm{As}_{2}, \mathrm{LiFeAs}$, FeSe were performed using the spectrometer described in Refs. [14]. The measurements of LaFeAsO were performed in a back-scattering geometry using a T64000 triple-stage spectrometer.

The phononic Raman scattering intensity is proportional to $I \propto\left|\hat{e}_{i} \cdot \mathbf{R} \cdot \hat{e}_{s}\right|^{2}$, where $\hat{e}_{i}$ and $\hat{e}_{s}$ are the polarization unit vectors of the incoming and scattering light, respectively, and $\mathbf{R}$ is the Raman tensor [29]. For the $D_{4 h}$ point group the $X X, X Y, X^{\prime} X^{\prime}$, and $X^{\prime} Y^{\prime}$ polarization geometries probe $A_{1 g}+B_{1 g}, A_{2 g}+B_{2 g}, A_{1 g}+B_{2 g}$, and $A_{2 g}+B_{1 g}$ symmetry excitations, respectively. In the orthorhombic phase with $D_{2 h}$ point group symmetry, the unit cell rotates by $45^{\circ}$; the $A_{1 g}$ and $B_{2 g}$ representations of the $D_{4 h}$ point group merge into the $A_{g}$ representation of the $D_{2 h}$ point group, and $A_{2 g}$ and $B_{1 g}\left(D_{4 h}\right)$ merge into $B_{1 g}\left(D_{2 h}\right)$. In the orthorhombic phase, the $X X$ and $X Y$ polarization geometries probe $A_{g}+B_{1 g}$ and $A_{g}$ symmetry excitations, respectively [14].

Before investigating the behavior of the $A_{1 g} / A_{g}$ symmetry As phonon across the magnetostructural transitions, we first examine the $A_{1 g}$ and $A_{g}$ Raman tensors:

$$
A_{1 g}^{D_{4 h}}=\left(\begin{array}{ccc}
\bar{a} & 0 & 0 \\
0 & \bar{a} & 0 \\
0 & 0 & \bar{c}
\end{array}\right), \quad A_{g}^{D_{2 h}}=\left(\begin{array}{ccc}
\frac{\left(\bar{a}^{\prime}+\bar{b}^{\prime}\right)}{2} & \frac{\left(\bar{a}^{\prime}-\bar{b}^{\prime}\right)}{2} & 0 \\
\frac{\left(\bar{a}^{\prime}-\bar{b}^{\prime}\right)}{2} & \frac{\left(\bar{a}^{\prime}+\bar{b}^{\prime}\right)}{2} & 0 \\
0 & 0 & \bar{c}
\end{array}\right),
$$

where $A_{g}^{D_{2 h}}$ (orthorhombic phase) has been rotated by $45^{\circ}$ to keep the same $X Y Z$ axis notation as in the tetragonal phase. Here, $\bar{a}^{\prime}$ and $\bar{b}^{\prime}$ are the diagonal elements of the $A_{g}^{D_{2 h}}$ Raman tensor in the natural coordinate system of the orthorhombic phase (before the $45^{\circ}$ rotation).

Accordingly, the $A_{1 g}$-symmetry mode is forbidden in the $X Y$ scattering geometry in the tetragonal phase. This is the case for LiFeAs, which shows neither structural nor magnetic transition. As shown in Fig. 2(a), sharp Raman phonon peaks at $186 \mathrm{~cm}^{-1}$ and $237 \mathrm{~cm}^{-1}$, corresponding to a $A_{1 g}($ As $)$ and a $B_{1 g}(\mathrm{Fe})$ modes, respectively, are detected in the $X X$ scattering geometry. However, as expected for the tetragonal structure of LiFeAs, these modes have no intensity in the $X Y$ scattering geometry. Similar Raman results are reported for the tetragonal $\mathrm{Fe}_{1+y} \mathrm{Te}_{0.6} \mathrm{Se}_{0.4}$ single crystal [30].

If anisotropy develops in the orthorhombic phase, the $A_{g}$ anion mode may acquire a finite intensity $\left|\left(\bar{a}^{\prime}-\bar{b}^{\prime}\right) / 2\right|^{2}$ in the $X Y$ scattering geometry related to the anisotropy of the inplane polarizability associated to this $A_{g}$ anion mode, because $\bar{a}^{\prime}$ and $\bar{b}^{\prime}$ are the polarizability derivatives along the two $\mathrm{Fe}-\mathrm{Fe}$ orthogonal directions $\left(X^{\prime}\right.$ and $\left.Y^{\prime}\right)$ in the orthorhombic phase. Since the lattice orthorhombicity $\delta$ is small (Table I), the intensity due to geometrical anisotropy is expected to be weak. For example, for the FeSe material, which exhibits a structural phase transition at $90 \mathrm{~K}[31,32]$ but no long-range magnetic ordering, we observe a $A_{g}(\mathrm{Se})$ phonon at $180 \mathrm{~cm}^{-1}$ and a $B_{1 g}(\mathrm{Fe})$ phonon at $208 \mathrm{~cm}^{-1}$ for the $X X$ polarization [Fig. 2(b)]. Although the intensity of the $A_{g}(\mathrm{Se})$ phonon with the $X Y$ polarization is finite at $20 \mathrm{~K}$ [inset of Fig. 2(b)], it is only $2 \%$ of the corresponding intensity recorded for the $X X$ polarization (Table I) [13,33]. 

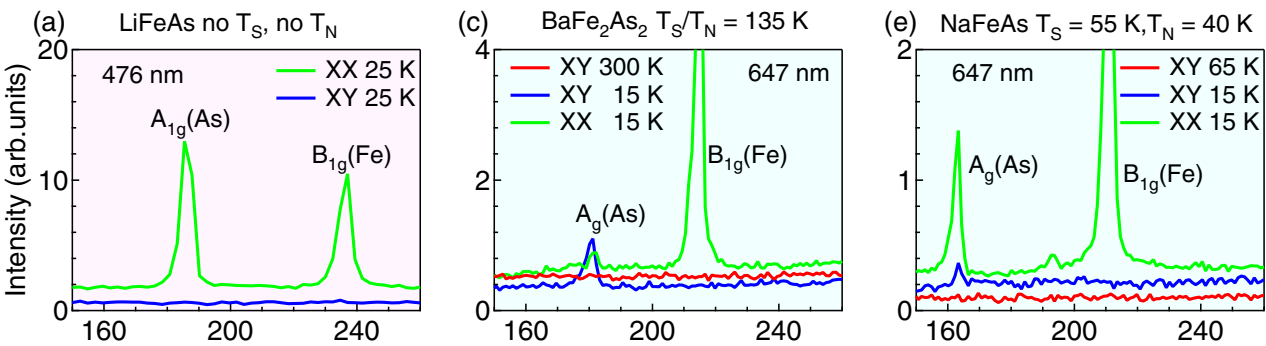

(b) $\quad \mathrm{FeSe}_{\mathrm{S}}=90 \mathrm{~K}, \mathrm{no} \mathrm{T}_{\mathrm{N}}$
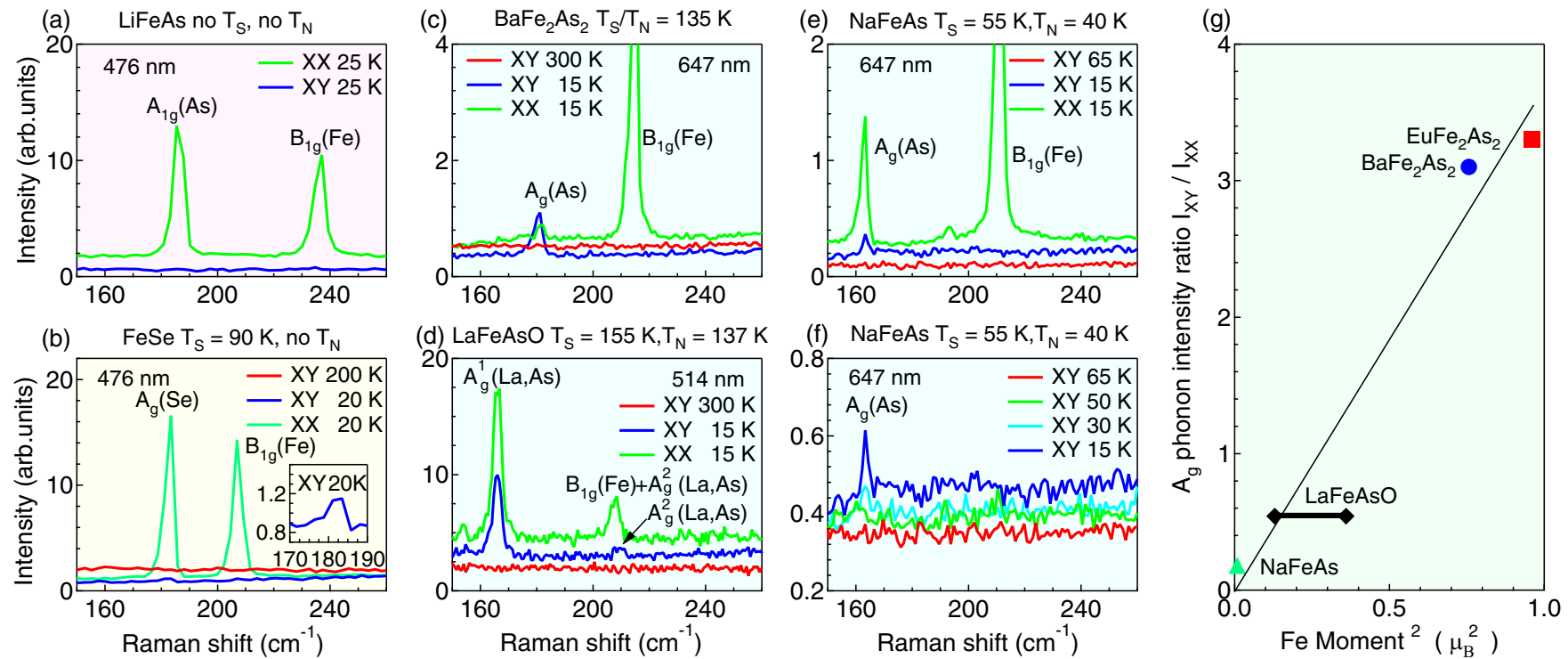

FIG. 2. Comparison of Raman spectra (shifted for clarity) in $X X$ and $X Y$ scattering geometries for different parent compounds: (a) LiFeAs, (b) FeSe, (c) $\mathrm{BaFe}_{2} \mathrm{As}_{2}$, (d) $\mathrm{LaFeAsO}$, (e) and (f) $\mathrm{NaFeAs}$. When finite, the $T_{N}$ and $T_{S}$ values are indicated on the top of the corresponding panel. (g) $A_{g}$ (As) phonon intensity ratio in $X Y$ vs. $X X$ geometries $\left(I_{X Y} / I_{X X}\right)$ at $15 \mathrm{~K}$ as a function of the squared ordered magnetic moment/Fe $[23,25]$. The black line is a linear fit.

In contrast, $\mathrm{BaFe}_{2} \mathrm{As}_{2}$ with strong magnetic ordering clearly shows the $181 \mathrm{~cm}^{-1} A_{g}$ (As) mode $[13,15,34,35]$ in the $X Y$ scattering geometry below $T_{N}$ [Fig. 2(c)]. Similar observation is made for NaFeAs [Figs. 2(e) and 2(f)], which also encounters both a structural and a magnetic phase transition: (i) We observe only a weak intensity between $T_{S}$ and $T_{N}$, and (ii) the $162 \mathrm{~cm}^{-1} A_{g}$ (As) phonon mode appears in the $X Y$ spectra only below $T_{N}$. LaFeAsO [36-38] is another system with split structural and magnetic phase transitions. In this case as well, we detect sizable intensity for the $A_{g}^{1}$ (in-phase La and As) mode at $166 \mathrm{~cm}^{-1}$ and the $A_{g}^{2}$ (out-of-phase La and As) mode at $209 \mathrm{~cm}^{-1}$ in the $X Y$ scattering geometry below $T_{N}$ [Fig. 2(d)].

To quantify the intensity of the $A_{g}$ (As) phonon in the $X Y$ scattering geometry below $T_{N}$ in different families of Fe-based superconductors, we study the ratio between the $A_{g}$ (As) mode intensity in the $X Y$ and $X X$ scattering geometries $I_{X Y} / I_{X X}$. This ratio is proportional to $\left|\left(\bar{a}^{\prime}-\bar{b}^{\prime}\right) /\left(\bar{a}^{\prime}+\bar{b}^{\prime}\right)\right|^{2}$, which is a direct measure of the in-plane polarizability anisotropy of the $A_{g}$ (As) mode. Based on Table I, the ratio $I_{X Y} / I_{X X}$ is significant only for compounds with long-range magnetic ordering. For example, the ratio $I_{X Y} / I_{X X}$ is $300 \%$ for $\mathrm{BaFe}_{2} \mathrm{As}_{2}, 16 \%$ for $\mathrm{NaFeAs}$ and $50 \%$ for $\mathrm{LaFeAsO}$, as compared to $2 \%$ for $\mathrm{FeSe}$, i.e., 1-2 orders of magnitude smaller. Such behavior cannot be solely explained by weak geometrical lattice orthorhombicity $\delta$, instead, the observation relates the mode's intensity to magnetic order parameter. This is best evident from Fig. 2(g), where we show that the $I_{X Y} / I_{X X}$ ratio of the $A_{g}$ (As) phonon intensity for different Fe-based families is proportional to the square of the magnetic moment $M^{2}$.

In conclusion, we revealed a significant intensity enhancement of the emergent $A_{g}$ (As) phonon mode in the $X Y$ scattering geometry below $T_{N}$ only for parent compounds of Fe-based superconductors showing magnetic order. We demonstrate that the ratio of the As phonon intensity in the $X Y$ and $X X$ scattering geometries $I_{X Y} / I_{X X}$ is proportional to the square of the magnetic ordered moment $M^{2}$. We conclude that the generic $A_{g}(\mathrm{As})$ phonon intensity enhancement below $T_{N}$ in iron pnictides is due to the in-plane electronic anisotropy induced by the collinear SDW order: a larger ordered moment in the magnetic phase results in lager in-plane electronic anisotropy, which in turn cause larger As phonon intensity ratio $I_{X Y} / I_{X X}$ below $T_{N}$.

We thank E. Bascones and K. Haule for discussions. The spectroscopic work conducted at Rutgers (S.-F.W., W.-L.Z., V.K.T., and G.B.) was supported by NSF Grant No. DMR-1709161. The sample preparation and characterization at ORNL (A.S.S.) was supported by the US Department of Energy, Basic Energy Sciences, Materials Sciences and Engineering Division. The work at NICPB was supported by the Estonian Research Council Grant No. PRG736 and by the European Research Council (ERC) under Grant Agreement No. 885413.
[1] R. M. Fernandes, A. V. Chubukov, and J. Schmalian, What drives nematic order in iron-based superconductors? Nature Phys. 10, 97 (2014).
[2] R. M. Fernandes, L. H. VanBebber, S. Bhattacharya, P. Chandra, V. Keppens, D. Mandrus, M. A. McGuire, B. C. Sales, A. S. Sefat, and J. Schmalian, Effects of Nematic Fluctuations 
on the Elastic Properties of Iron Arsenide Superconductors, Phys. Rev. Lett. 105, 157003 (2010).

[3] W. Z. Hu, J. Dong, G. Li, Z. Li, P. Zheng, G. F. Chen, J. L. Luo, and N. L. Wang, Origin of the Spin Density Wave Instability in $\mathrm{AFe}_{2} \mathrm{As}_{2}(\mathrm{~A}=\mathrm{Ba}, \mathrm{Sr})$ as Revealed by Optical Spectroscopy, Phys. Rev. Lett. 101, 257005 (2008).

[4] Y. Ran, F. Wang, H. Zhai, A. Vishwanath and D.-H. Lee, Nodal spin density wave and band topology of the FeAs-based materials, Phys. Rev. B 79, 014505 (2009).

[5] P. Richard, K. Nakayama, T. Sato, M. Neupane, Y.-M. Xu, J. H. Bowen, G. F. Chen, J. L. Luo, N. L. Wang, X. Dai, Z. Fang, H. Ding and T. Takahashi, Observation of Dirac Cone Electronic Dispersion in $\mathrm{BaFe}_{2} \mathrm{As}_{2}$, Phys. Rev. Lett. 104, 137001 (2010).

[6] W.-L. Zhang, Z. P. Yin, A. Ignatov, Z. Bukowski, Janusz Karpinski, Athena S. Sefat, H. Ding, P. Richard, and G. Blumberg, Raman scattering study of spin-density-waveinduced anisotropic electronic properties in $\mathrm{AFe}_{2} \mathrm{As}_{2}(\mathrm{~A}=\mathrm{Ca}$, Eu), Phys. Rev. B 93, 205106 (2016).

[7] J.-H. Chu, J. G. Analytis, K. De Greve, P. L. McMahon, Z. Islam, Y. Yamamoto, and I. R. Fisher, In-plane resistivity anisotropy in an underdoped iron arsenide superconductor, Science 329, 824 (2010).

[8] A. Dusza, A. Lucarelli, F. Pfuner, J.-H. Chu, I. R. Fisher, and L. Degiorgi, Anisotropic charge dynamics in detwinned $\mathrm{Ba}\left(\mathrm{Fe}_{1-x} \mathrm{Co}_{x}\right)_{2} \mathrm{As}_{2}$, Europhys. Lett. 93, 37002 (2011).

[9] M. Nakajima, T. Liang, S. Ishida, Y. Tomioka, K. Kihou, C. H. Lee, A. Iyo, H. Eisaki, T. Kakeshita, T. Ito, and S. Uchida, Unprecedented anisotropic metallic state in undoped iron arsenide $\mathrm{BaFe}_{2} \mathrm{As}_{2}$ revealed by optical spectroscopy, Proc. Natl. Acad. Sci. USA 108, 12238 (2011).

[10] S. Jiang, H. S. Jeevan, J. K. Dong, and P. Gegenwart, Thermopower As a Sensitive Probe of Electronic Nematicity in Iron Pnictides, Phys. Rev. Lett. 110, 067001 (2013).

[11] E. P. Rosenthal, E. F. Andrade, C. J. Arguello, R. M. Fernandes, L. Y. Xing, X. C. Wang, C. Q. Jin, A. J. Millis, and A. N. Pasupathy, Visualization of electron nematicity and unidirectional antiferroic fluctuations at high temperatures in NaFeAs, Nature Phys. 10, 225 (2014).

[12] M. Yi, D. Lu, J.-H. Chu, J. G. Analytis, A. P. Sorini, A. F. Kemper, B. Moritz, S.-K. Mo, R. G. Moore, M. Hashimoto, W.-S. Lee, Z. Hussain, T. P. Devereaux, I. R. Fisher, and Z.-X. Shen, Symmetry-breaking orbital anisotropy observed for detwinned $\mathrm{Ba}\left(\mathrm{Fe}_{1-x} \mathrm{Co}_{x}\right)_{2} \mathrm{As}_{2}$ above the spin density wave transition, Proc. Natl. Acad. Sci. USA 108, 6878 (2011).

[13] A. Baum, Y. Li, M. Tomić, N. Lazarević, D. Jost, F. Löffler, B. Muschler, T. Böhm, J.-H. Chu, I. R. Fisher, R. Valentí, I. I. Mazin, and R. Hackl, Interplay of lattice, electronic, and spin degrees of freedom in detwinned $\mathrm{BaFe}_{2} \mathrm{As}_{2}$ : A Raman scattering study, Phys. Rev. B 98, 075113 (2018).

[14] S.-F. Wu, W.-L. Zhang, L. Li, H.-B. Cao, H.-H. Kung, A. S. Sefat, H. Ding, P. Richard, and G. Blumberg, Coupling of fully symmetric As phonon to magnetism in $\mathrm{Ba}\left(\mathrm{Fe}_{1-x} \mathrm{Au}_{x}\right)_{2} \mathrm{As}_{2}$, Phys. Rev. B 102, 014501 (2020).

[15] F. Kretzschmar, T. Bohm, U. Karahasanovic, B. Muschler, A. Baum, D. Jost, J. Schmalian, S. Caprara, M. Grilli, C. Di Castro, J. G. Analytis, J.-H. Chu, I. R. Fisher, and R. Hackl, Critical spin fluctuations and the origin of nematic order in $\mathrm{Ba}\left(\mathrm{Fe}_{1-x} \mathrm{Co}_{x}\right)_{2} \mathrm{As}_{2}$, Nature Phys. 12, 560 (2016).

[16] A. S. Sefat, Bulk synthesis of iron-based superconductors, Curr. Opin. Solid State Mater. Sci. 17, 59 (2013).
[17] L. Li, H. B. Cao, M. A. McGuire, J. S. Kim, G. R. Stewart, and A. S. Sefat, Role of magnetism in superconductivity of $\mathrm{BaFe}_{2} \mathrm{As}_{2}$ : Study of $5 d$ Au-doped crystals, Phys. Rev. B 92, 094504 (2015).

[18] M. A. Tanatar, N. Spyrison, K. Cho, E. C. Blomberg, G. Tan, P. Dai, C. Zhang, and R. Prozorov, Evolution of normal and superconducting properties of single crystals of $\mathrm{Na}_{1-\delta} \mathrm{FeAs}$ upon interaction with environment, Phys. Rev. B 85, 014510 (2012).

[19] Y. Kamihara, T. Watanabe, M. Hirano, and H. Hosono, Ironbased layered superconductor $\mathrm{LaO}_{1-x} \mathrm{~F}_{x} \mathrm{FeAs}$ ( $\mathrm{x}=0.05-$ 0.12) with $T_{c}=26 \mathrm{~K}$, J. Am. Chem. Soc 130, 3296 (2008).

[20] C. de la Cruz, Q. Huang, J. W. Lynn, J. Li, W. R. II, J. L. Zarestky, H. A. Mook, G. F. Chen, J. L. Luo, N. L. Wang, and P. C Dai, Magnetic order close to superconductivity in the iron-based layered $\mathrm{LaO}_{1-x} \mathrm{~F}_{x} \mathrm{FeAs}$ systems, Nature (London) 453, 899 (2008).

[21] S. Hosoi, K. Matsuura, K. Ishida, H. Wang, Y. Mizukami, T. Watashige, S. Kasahara, Y. Matsuda, and T. Shibauchi, Nematic quantum critical point without magnetism in $\mathrm{FeSe}_{1-x} \mathrm{~S}_{x}$ superconductors, Proc. Natl. Acad. Sci. USA 113, 8139 (2016).

[22] M. Tegel, M. Rotter, V. Weiß, F. M. Schappacher, R. Pöttgen, and D. Johrendt, Structural and magnetic phase transitions in the ternary iron arsenides $\mathrm{SrFe}_{2} \mathrm{As}_{2}$ and $\mathrm{EuFe}_{2} \mathrm{As}_{2}$, J. Phys.: Condens. Matter 20, 452201 (2008).

[23] Y. Xiao, Y. Su, M. Meven, R. Mittal, C. M. N. Kumar, T. Chatterji, S. Price, J. Persson, N. Kumar, S. K. Dhar, A. Thamizhavel, and Th. Brueckel, Magnetic structure of $\mathrm{EuFe}_{2} \mathrm{As}_{2}$ determined by single-crystal neutron diffraction, Phys. Rev. B 80, 174424 (2009).

[24] Q. Huang, Y. Qiu, W. Bao, M. A. Green, J. W. Lynn, Y. C. Gasparovic, T. Wu, G. Wu, and X. H. Chen, NeutronDiffraction Measurements of Magnetic Order and a Structural Transition in the Parent $\mathrm{BaFe}_{2} \mathrm{As}_{2}$ Compound of FeAsBased High-Temperature Superconductors, Phys. Rev. Lett. 101, 257003 (2008).

[25] P. C. Dai, Antiferromagnetic order and spin dynamics in ironbased superconductors, Rev. Mod. Phys. 87, 855 (2015).

[26] S. Li, C. de la Cruz, Q. Huang, G. F. Chen, T.-L. Xia, J. L. Luo, N. L. Wang, and P. C Dai, Structural and magnetic phase transitions in $\mathrm{Na}_{1-\delta} \mathrm{FeAs}$, Phys. Rev. B 80, 020504(R) (2009).

[27] T. M. McQueen, A. J. Williams, P. W. Stephens, J. Tao, Y. Zhu, V. Ksenofontov, F. Casper, C. Felser, and R. J. Cava, Tetragonal-to-Orthorhombic Structural Phase Transition at $90 \mathrm{~K}$ in the Superconductor $\mathrm{Fe}_{1.01} \mathrm{Se}$, Phys. Rev. Lett. 103 , 057002 (2009).

[28] X. C. Wang, Q. Q. Liu, Y. X. Lv, Z. Deng, K. Zhao, R. C. Yu, J. L. Zhu, and C. Q Jin, Superconducting properties of "111" type LiFeAs iron arsenide single crystals, Sci. China Phys. Mech. 53, 1199 (2010).

[29] M. V. Klein, Electronic Raman Scattering, in Light Scattering in Solids I, Vol. 8, edited by M. Cardona (Springer-Verlag, Berlin, 1983), Chap. 4, pp. 147-202.

[30] S.-F. Wu, A. Almoalem, I. Feldman, A. Lee, A. Kanigel, and G. Blumberg, Superconductivity and phonon self-energy effects in $\mathrm{Fe}_{1+y} \mathrm{Te}_{0.6} \mathrm{Se}_{0.4}$, Phys. Rev. Research 2, 013373 (2020).

[31] A. E. Böhmer, F. Hardy, F. Eilers, D. Ernst, P. Adelmann, P. Schweiss, T. Wolf, and C. Meingast, Lack of coupling between superconductivity and orthorhombic distortion in stoichiometric single-crystalline FeSe, Phys. Rev. B 87, 180505(R) (2013). 
[32] A. E. Böhmer, T. Arai, F. Hardy, T. Hattori, T. Iye, T. Wolf, H. v. Löhneysen, K. Ishida, and C. Meingast, Origin of the Tetragonal-to-Orthorhombic Phase Transition in FeSe: A Combined Thermodynamic and NMR Study of Nematicity, Phys. Rev. Lett. 114, 027001 (2015).

[33] W.-L. Zhang, S.-F. Wu, S. Kasahara, T. Shibauchi, Y. Matsuda, and G. Blumberg, arXiv:1710.09892.

[34] L. Chauvière, Y. Gallais, M. Cazayous, A. Sacuto, M. A. Méasson, D. Colson, and A. Forget, Doping dependence of the lattice dynamics in $\mathrm{Ba}\left(\mathrm{Fe}_{1-x} \mathrm{Co}_{x}\right)_{2} \mathrm{As}_{2}$ studied by Raman spectroscopy, Phys. Rev. B 80, 094504 (2009).

[35] S. Sugai, Y. Mizuno, R. Watanabe, T. Kawaguchi, K. Takenaka, H. Ikuta, Y. Takayanagi, N. Hayamizu, and Y. Sone, Spin- density-wave gap with dirac nodes and two-magnon Raman scattering in $\mathrm{BaFe}_{2} \mathrm{As}_{2}$, J. Phys. Soc. Jpn. 81, 024718 (2012).

[36] U. F. Kaneko, P. F. Gomes, A. F. García-Flores, J.-Q. Yan, T. A. Lograsso, G. E. Barberis, D. Vaknin, and E. Granado, Nematic fluctuations and phase transitions in LaFeAsO: A Raman scattering study, Phys. Rev. B 96, 014506 (2017).

[37] V. G. Hadjiev, M. N. Iliev, K. Sasmal, Y.-Y. Sun, and C. W. Chu, Raman spectroscopy of $R \mathrm{FeAsO}$ ( $R=\mathrm{Sm}, \mathrm{La})$, Phys. Rev. B 77, 220505(R) (2008).

[38] S. C. Zhao, D. Hou, Y. Wu, T. L. Xia, A. M. Zhang, G. F. Chen, J. L. Luo, N. L. Wang, J. H. Wei, Z. Y. Lu, and Q. M. Zhang, Raman spectra in iron-based quaternary $\mathrm{CeO}_{1-x} \mathrm{~F}_{x} \mathrm{FeAs}$ and $\mathrm{LaO}_{1-x} \mathrm{~F}_{x} \mathrm{FeAs}$, Supercond. Sci. Tech 22, 015017 (2008). 